\title{
Locked Plate Fixation of Osteoporotic Humeral Shaft Fractures: Are Two Locking Screws Per Segment Enough?
}

\author{
David J. Hak, MD, MBA, Peter Althausen, MD, MBA, and Scott J. Hazelwood, PhD
}

\begin{abstract}
Objective: The purpose of this study was to compare the biomechanical behavior of using two versus three locking screws per bone segment in a cadaveric humerus fracture gap model.
\end{abstract}

Methods: Six matched pairs of elderly osteoporotic fresh-frozen human cadaveric humerii were used. An eight-hole locking compression plate was placed posteriorly on the humeral shaft and secured with either four or six bicortical locking screws. A 5-mm middiaphyseal gap osteotomy was created to simulate a comminuted fracture without bony contact. Specimens were tested in offset axial compression, four-point anteroposterior bending, four-point mediallateral bending, and torsion. After the initial testing in each of these modalities, the constructs were cyclically loaded in torsion and again tested in the four loading modalities. Lastly, the fixation constructs were then tested to failure in torsion.

Results: There were no significant differences in stiffness between the group fixed with two screws per segment and the group fixed with three screws per segment. The peak torque to failure was higher in the four-screw construct compared with the six-screw construct. The mean torque to failure was $23.5 \pm 3.7 \mathrm{Nm}$ in the construct with two locking screws per segment compared with $20.4 \pm 2.8 \mathrm{Nm}$ in the construct with three locking screws per segment $(P=0.030)$.

Conclusions: The addition of a third screw in the locked plate construct did not add to the mechanical stability in axial loading, bending, or torsion. In testing to failure, the addition of a third screw resulted in lower load to failure.

Key Words: locking plate, humerus shaft fracture, biomechanics, osteoporosis

\section{INTRODUCTION}

Humeral shaft fractures are relatively common injuries in the aging population. ${ }^{1}$ The majority of humeral shaft fractures can be treated nonoperatively with high union rates and good functional results. ${ }^{2}$ In cases in which internal fixation is indicated, plate fixation results in a high union rate and a low incidence of complications. ${ }^{3}$

Osteoporosis compromises screw fixation and leads to increased failure rate after plate fixation. In fractures initially treated nonoperatively that fail to heal, disuse leads to increased osteopenia further compromising screw fixation. Ring et al have demonstrated that nonunions of the upper extremity in the elderly are extremely disabling. ${ }^{4}$ These individuals are less able to adapt to changes and depend on an intact upper extremity to maintain function and independence in activities of daily living.

Locking plate fixation is being increasingly used for the treatment of fractures in patients with osteoporosis; however, questions remain about how many locking screws are necessary to achieve adequate fixation. The purpose of this biomechanical study was to examine the resistance to displacement of paired humerii with transverse gap osteotomies that have been internally stabilized with a locking plate fixed with either two or three bicortical locking screws per fracture segment.

\section{MATERIALS AND METHODS}

Fresh matched cadaveric humerii pairs were harvested from six donors that included three males and three females ranging in age from 75 to 87 years with a mean of 82 years. Specimens were cleaned of soft tissues, covered in salinesoaked gauze, and frozen to $-20^{\circ} \mathrm{C}$ until preparation and testing. The prepared specimens were kept moist with normal saline during testing. Specimens were imaged by standard radiographs and dual energy x-ray absorptiometry (DXA). For DXA scanning, a standard region of interest $40 \mathrm{~cm}$ long centered on the middiaphysis was selected and the bone mineral density $\left(\mathrm{g} / \mathrm{cm}^{2}\right)$ was calculated using software from the manufacturer (Hologic, Inc., Bedford, MA). An estimation of the patient's T-score was calculated by comparing the calculated humeral shaft bone mineral density with standard values for young adult forearms.

The proximal and distal ends of the humerii were potted colinearly within methylmethacrylate blocks to provide a substrate for securing the bone during testing. Mechanical testing was performed in a manner previously described in the literature. ${ }^{5}$ Each pair of humerii was initially tested 
nondestructively on an Instron (Norwood, MA) mechanical testing machine. Baseline load versus deformation curves were obtained for the intact humerii using three loading modalities; offset axial compression, four-point bending (in both posterior-anterior and lateral-medial directions), and torsion. Stiffness of the construct was determined from the linear portion of the load deformation curve.

A test fixture with the upper loading supports spaced $5 \mathrm{~cm}$ apart and the lower loading supports spaced $15 \mathrm{~cm}$ apart was used for four-point bending. Loads with a maximum force of $200 \mathrm{~N}$ were applied to the humerii in a lateral-medial and posterior-anterior direction. Offset axial loading was performed by securing the distal potted block in a custom fixture and loading the proximal humerus $4 \mathrm{~cm}$ medial to its central axis. A maximum force of $100 \mathrm{~N}$ was applied to simulate an inferiorly directed vertical force on the humeral head surface. Torsion was tested by applying a maximum load of $4.5 \mathrm{Nm}$ in external rotation along the central longitudinal axis of the humerus. During torsional testing, the specimens were held rigidly without an applied axial load.

The humerii were then cycled in torsion between 0 and $4.5 \mathrm{Nm}$ at $1 \mathrm{~Hz}$ for 1000 cycles to simulate upper extremity use during the early postoperative period and then retested in the three loading modalities to obtain load versus deformation curves.

Right and left paired humerii were randomly instrumented with an eight-hole narrow large-fragment locking plate (Synthes, Paoli, PA). The plate was positioned on the posterior aspect of the humeral shaft and centered at the middiaphyseal level. A locking drill sleeve was threaded into the plate and a 4.3-mm diameter drill bit used to drill a hole at right angles to the long axis of the plate. Five-millimeter diameter locking screws were inserted and tightened to a maximum torque of 3.4 Nm. Screws longer than would be chosen clinically were used to ensure that the far cortex was fully engaged by the threaded portion of the screw. Paired humerii were instrumented with either two bicortical screws (placed in holes 1, 3, 6, and 8), or three bicortical screws (placed in holes 1, 2, 3, 6, 7, and 8) per fracture segment. A 5-mm transverse gap osteotomy was then created with a hand saw at the midpoint of the plate to simulate a comminuted fracture without cortical bone contact (Fig. 1).

The specimens were then tested nondestructively in the manner described previously. Finally, each humerus was

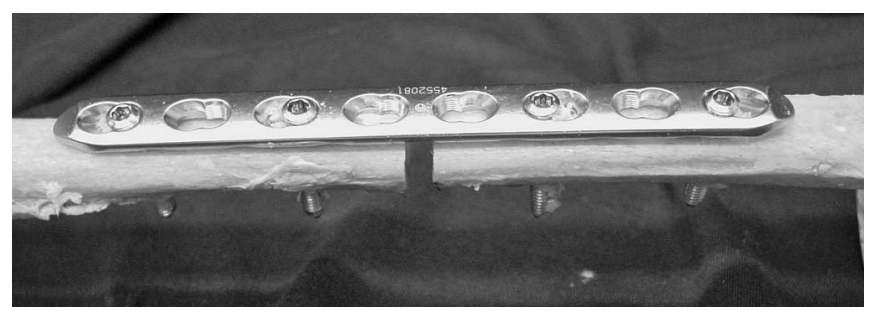

FIGURE 1. Five-millimeter transverse osteotomy gap in a specimen fixed with two bicortical locking screws per segment (screws placed in holes 1, 3, 6, and 8). In the contralateral humerus, a third bicortical screw was placed in each segment (screws placed in holes 1, 2, 3, 6, 7, and 8). loaded until failure in torsion to determine the maximum torque to failure. The site and pattern of fixation failure were recorded.

The resistance to displacement was determined by the slope of the load versus deformation curves for the two constructs under the three loading sequences before and after cycling. Statistical analyses were performed on both the stiffness values and on normalized stiffness values expressed as a percentage of the intact stiffness, which were obtained before instrumentation to minimize any differences between right- versus left-sided variations. An analysis of variance was performed to determine differences between the measured stiffness values and normalized stiffness values for each plate at each loading modality before and after cyclic loading ( $P<0.05$ significant $)$. In addition, statistical analyses were performed using paired $t$ tests to determine the significance of any differences between the two plates in stiffness before cyclic loading, stiffness after cyclic loading, and maximum torque to failure $(P<0.05$ significant).

\section{RESULTS}

Humerii ranged in length from 28 to $34 \mathrm{~cm}$ with a mean of $32 \mathrm{~cm}$. Pretesting radiographs demonstrated no evidence of fracture or pathologic lesion in any specimen. DXA scanning was consistent with osteoporotic bone in all specimens with estimated T-scores ranging from -2.3 to -4.9 with a mean of -3.7. Two-tailed $t$ test demonstrated no inherent right-left differences in the intact samples before plating.

There were no significant differences between the precycle stiffness or normalized stiffness and those after 1000 cycles of physiological loading (Table 1, Figs. 2-5). Specimens were least stiff relative to the intact bone in anteroposterior bending. Bending in this direction corresponds to the plate's smallest moment of inertia (thinnest crosssection). Constructs were stiffest in medial-lateral bending. Bending in this direction corresponds to the plate's largest moment of inertia (thickest portion of the plate). In addition, constructs tested in medial-lateral bending were significantly stiffer than their corresponding intact values (Table 1).

TABLE 1. Summary of Mean Stiffness Values ( \pm Standard Deviations) in Different Modes of Mechanical Testing

\begin{tabular}{lcc}
\hline Loading Mode & $\begin{array}{c}\text { Two Screws } \\
\text { per Segment }\end{array}$ & $\begin{array}{r}\text { Three Screws } \\
\text { per Segment }\end{array}$ \\
\hline Axial loading (N/mm) Intact stiffness: $602 \pm 290$ & \\
Before cycling & $444 \pm 210$ & $454 \pm 128$ \\
After cycling & $509 \pm 196$ & $578 \pm 296$ \\
Torsional loading (N-mm/degree) Intact stiffness: $682 \pm 131$ & \\
Before cycling & $498 \pm 121$ & $553 \pm 95$ \\
After cycling & $492 \pm 74$ & $606 \pm 181$ \\
Four-point anteroposterior bending $(\mathrm{N} / \mathrm{mm})$ Intact stiffness: $489 \pm 202$ \\
Before cycling & $101 \pm 39$ & $132 \pm 41$ \\
After cycling & $104 \pm 43$ & $111 \pm 17$ \\
Four-point medial-lateral bending $(\mathrm{N} / \mathrm{mm})$ Intact stiffness: $424 \pm 145$ \\
Before cycling & $521 \pm 67$ & $571 \pm 90$ \\
After cycling & $555 \pm 50$ & $593 \pm 58$ \\
\hline
\end{tabular}




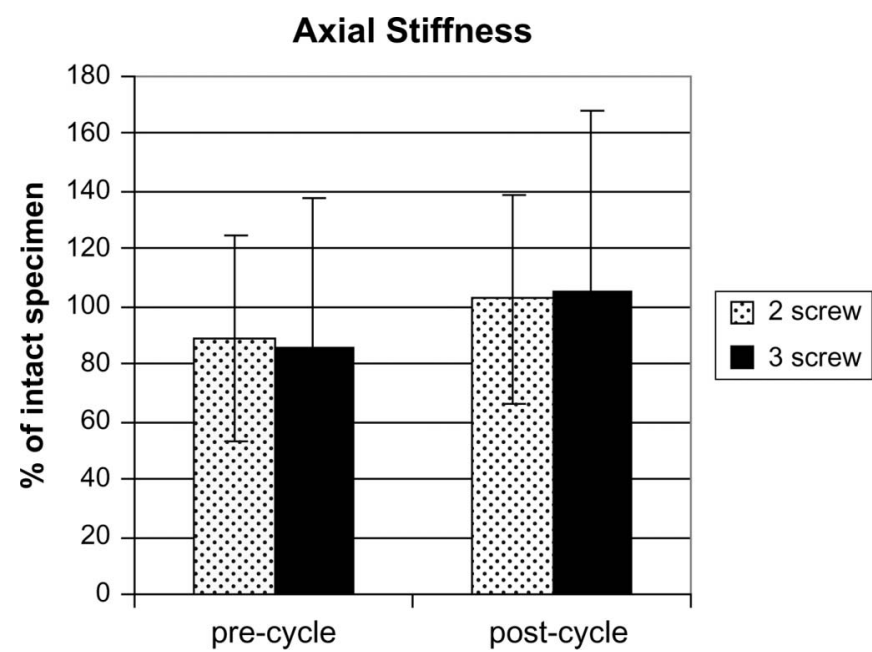

FIGURE 2. Normalized axial load testing showed no significant difference in stiffness between humerii instrumented with two locking screws per fragment versus three locking screws per segment.

There were no significant differences in stiffness or normalized stiffness between the group fixed with two screws per segment and the group fixed with three screws per segment for any loading modalities before or after cyclic testing. Similar values were seen in all modes of testing (Figs. 2-5).

The peak torque to failure was higher in the four-screw construct compared with the six-screw construct. The mean peak load was $23.5 \pm 3.7 \mathrm{Nm}$ in the four-screw constructs and was actually less, $20.4 \pm 2.8 \mathrm{Nm}$, in the six-screw constructs. A paired $t$ test revealed a $P$ value of 0.030 .

All final failures in the four-screw constructs consisted of spiral fractures through the distal screw hole. Spiral fractures also occurred in the six-screw constructs with four occurring through the distal screw hole and two

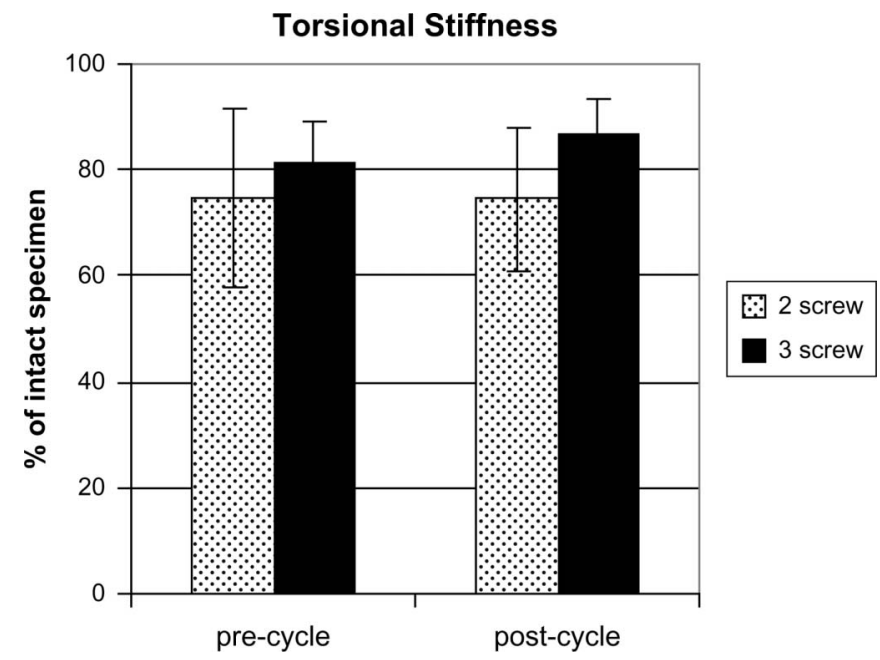

FIGURE 3. Normalized torsional load testing showed no significant difference in stiffness between humerii instrumented with two locking screws per fragment versus three locking screws per segment.

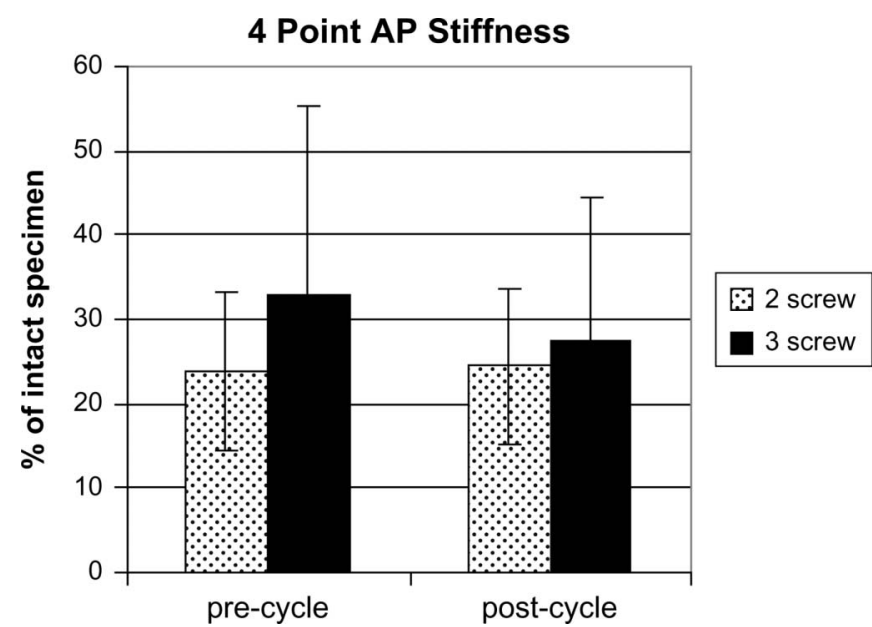

FIGURE 4. Normalized four-point anteroposterior bending showed no significant difference in stiffness between humerii instrumented with two locking screws per fragment versus three locking screws per segment.

through the middle screw hole. There were no cases of screw pullout.

\section{DISCUSSION}

When using locked plate fixation, the number of screws and the number of cortices needed per segment continues to be a topic of debate. In this study, we found no mechanical advantage for using more than two locking screws per bone segment in an osteoporotic humerus fracture gap model. In this gap osteotomy model, the mechanical properties of the construct appeared to be most influenced by the mechanical properties of the plate between the two inner locking screws. The distance between the two inner screws was not altered between the two groups. The torque to failure was actually less

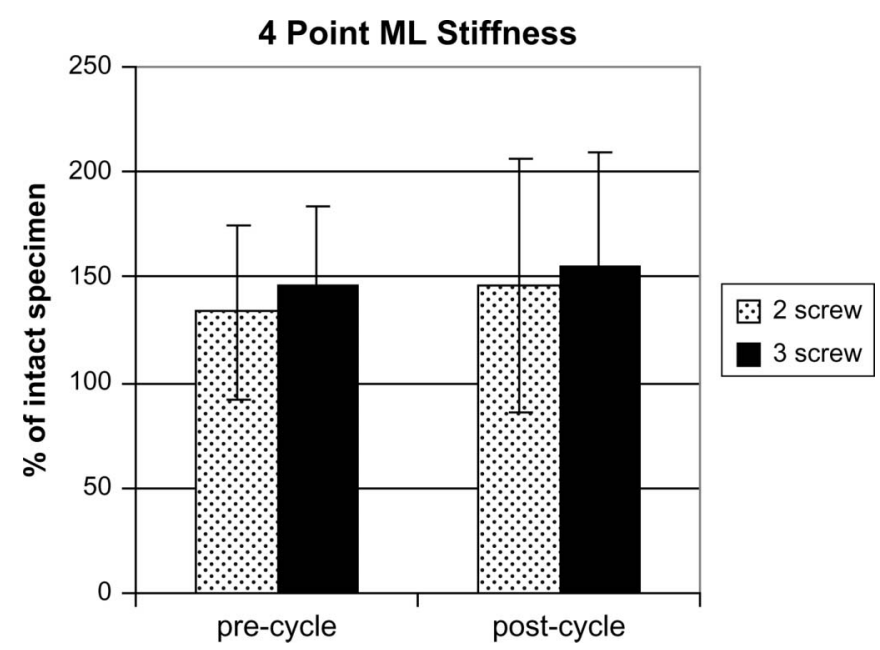

FIGURE 5. Normalized four- point medial-lateral bending showed no significant difference in stiffness between humerii instrumented with two locking screws per fragment versus three locking screws per segment. 
when three screws were used compared with two. The three closely spaced screws may create a linear stress riser leading to reduced resistance to fracture.

Authors have provided various recommendations for the minimum number of locking screws and the minimum number of cortices required per segment. Based on the clinical observation of radial and ulnar shaft radiolucencies at the bonescrew interface with the PC-Fix (Point Contact Fixator, Synthes, Paoli, PA) implant, which uses unicortical screw fixation, Hertel et al recommended obtaining at least three cortices per segment. ${ }^{6}$ In good quality bone, Gautier and Sommer have recommended using a minimum two screws per segment with at least three cortices for simple fractures and at least four cortices for comminuted fractures. In other cases such as osteoporotic bone, they have recommended a minimum of three screws per segment. ${ }^{7}$ In osteoporotic bone, Wagner has recommended the use of at least three locking screws in each main fragment with at least one of them being bicortical. ${ }^{8}$ Because rotational forces predominate in the upper extremity, Stoffel et al recommended the use of three or four locking screws in each main humerus fragment to improve torsional rigidity. ${ }^{9}$

Screw location and plate length may be as important as screw number, because it influences the construct stiffness and amount of motion seen at the fracture. Stoffel et al examined different screw configurations using a 12-hole $4.5-\mathrm{mm}$ titanium locking compression plate in composite synthetic bone cylinders. They found that axial stiffness and torsional rigidity were mainly influenced by the distance between the fracture site and the closest screw. Moving the screw one hole farther from the fracture the construct became almost twice as flexible in compression and torsion. ${ }^{9}$ Similar observations have been noted with nonlocking plate fixation. In a study using composite foam blocks, Törnkvist et al showed that the bending strength of screw-plate fixation can be increased by using a longer plate with screws spaced further apart. ${ }^{10}$ In a cadaveric ulnar osteotomy model, Sanders et al concluded that the length of the plate is more important than the number of screws and stated that once the working length (defined as the distance between the fracture and the nearest screw) is minimized and the plate length maximized, only two screws need to be inserted on each side of the fracture. ${ }^{11}$ Similarly, in our study using the same length plate, we found no advantage to the placement of a third locking screw in between two equally spaced screws.

Internal fixation of long bone fractures in the elderly patient is challenging secondary to the problems of osteoporotic bone. ${ }^{4}$ In osteoporotic bone, poor screw purchase results in sequential loosening of nonlocked screws and subsequent levering of the plate away from the bone. Loss of nonlocked screw purchase in osteoporotic bone is an important factor leading to failure of internal fixation of humeral shaft fracture fixation. The development of locking plates has provided an alternative to standard compression plates. Locking plates can provide fracture fixation without the undesirable effects on periosteal vascularization and mechanical drawbacks that are encountered with standard compression plates. ${ }^{12}$

In a retrospective clinical study comparing the use of locked plates and standard plates in the treatment of humeral nonunions and delayed unions, the authors suggested that locking plates may be a more reliable implant. There was one hardware failure in the 14 patients treated with standard plates but no failures in the 19 patients treated with a locked plate construct. ${ }^{13}$ Ring et al have reported on 24 patients with osteoporosis with humeral diaphyseal nonunions or delayed unions treated with a locking compression plate. ${ }^{14}$ Twenty-two of the patients united with the initial surgical treatment, whereas the remaining two that initially received demineralized bone healed after the addition of iliac crest bone graft. The authors noted no loss of fixation or implant breakage. They used a combination of standard screws to bring the plate to the bone, and locking screws, but did not specifically state the number of locking screws used per segment. Regardless of the number of locking screws used, it is important that locking screws are placed accurately, because deviation in the insertion angle greater than $5^{\circ}$ can significantly decrease the fixation stability. ${ }^{15}$

In standard plates, the biomechanical effect of different fixation constructs in torsion differs from that seen in bending. Törnkvist et al showed that in torsion, the fixation strength was dependent on the number of screws securing the plate. ${ }^{10} \mathrm{In}$ comparison, in the locking plate construct, we did not see an improvement in the fixation stability with the addition of a third screw per fracture segment. The locking plate eliminates screw pullout as a means of failure in torsion as demonstrated by our study.

One potential advantage of the locked plate construct is prevention of screw loosening through repeated loading. Although we did cycle the implants to simulate sequential load, we limited this to 1000 cycles to maintain the integrity of the cadaveric bone. No difference was observed between the precycle and postcycle testing results. This lack of effect has been noted by other authors during humeral biomechanical testing. ${ }^{16} \mathrm{It}$ is possible that a difference in pre- and postcycling stiffness in any modality may have been detected with greater numbers.

There are several limitations of the present study. Our model examined placement of only one length plate with specific screw configuration. The distance between the two inner most screws remained unchanged between the two groups. This screw position, which represents the plate working length, plays a critical role on the mechanical properties of the construct. Although DXA scanning was performed on these specimens obtained from an elderly population, this method does not necessarily correlate with standard hip, wrist, and spine DXA values. Microfractures can occur during nondestructive testing, especially in osteoporotic bone, and this may have influenced subsequent modes of mechanical testing. We did not perform any radiographic evaluation after mechanical testing, which may have identified such microfractures. Finally, a significant limitation of this study was the small sample size, resulting in statistical analysis with relatively weak power.

Biomechanics of the fixation device is only one of many factors to be considered in the operative treatment of humeral shaft fractures. Although numerous factors affect operative treatment decisions, it is worthwhile for the orthopaedic surgeon to have comparative biomechanical information of different plating constructs. Because the amount of motion at 
the fracture site influences the biologic reaction of bone, the biomechanical stability of a fracture fixation implant plays an important role in fracture healing.

\section{CONCLUSIONS}

In summary, we could not show a mechanical benefit from the addition of a third bicortical locking screw per fracture fragment in the manner tested. It is important to know that locking screws are substantially more expensive than standard screws. Although the cost of fixation failure and need for reoperation vastly exceed the total costs for an internal fixation implant, the indiscriminate use of unnecessary locking screws increases overall treatment costs. Clinically, whether two bicortical locking screws per fracture fragment provide sufficient fixation in comminuted osteoporotic humerus shaft fractures will need to be examined in future clinical studies.

\section{REFERENCES}

1. Baron JA, Karagas M, Barrett J, et al. Basic epidemiology of fractures of the upper end and lower limb among Americans over 65 years of age. Epidemiology. 1996;7:612-618.

2. Sarmiento A, Kinman PB, Galvin EG, et al. Functional bracing of fractures of the shaft of the humerus. J Bone Joint Surg Am. 1977;59: 596-601.

3. Gregory PR, Sanders RW. Compression plating versus intramedullary fixation of humeral shaft fractures. J Am Acad Orthop Surg. 1997;5: 215-223.

4. Ring, D, Perey, BH, Jupiter, JB. The functional outcome of ununited fractures of the humeral diaphysis in older patients. J Bone Joint Surg Am. 1999;81:177-190.
5. Jazrawi LM, Bai B, Simon JA, et al. A biomechanical comparison of Schuli nuts or cement augmented screws for plating of humeral fractures. Clin Orthop Relat Res. 2000;377:235-240.

6. Hertel R, Eijer H, Meisser A, et al. Biomechanical and biological considerations relating to the clinical use of the Point Contact-Fixatorevaluation of the device handling test in the treatment of diaphyseal fractures of the radius and/or ulna. Injury. 2001;32(Suppl 2):SB10-SB14.

7. Gautier E, Sommer C. Guidelines for the clinical application of the LCP. Injury. 2003;34(Suppl 2):B63-B76.

8. Wagner M. General principles for the clinical use of the LCP. Injury Int $J$ Care Injured. 2003;34:S-B31-S-B42.

9. Stoffel K, Dieter U, Stachowiak G, et al. Biomechanical testing of the LCP-how can stability in locked internal fixators be controlled? Injury Int $J$ Care Injured. 2003;34:S-B11-S-B19.

10. Törnkvist H, Hearn TC, Schatzker J. The strength of plate fixation in relation to the number and spacing of bone screws. J Orthop Trauma. 1996;10:204-208.

11. Sanders R, Haidukewych GJ, Milne T, et al. Minimal versus maximal plate fixation techniques of the ulna: the biomechanical effect of number of screws and plate length. $J$ Orthop Trauma. 2002;16:166-171.

12. Frigg, R. Locking compression plate (LCP). An osteosynthesis plate based on the dynamic compression plate and the point contact fixator (PC-Fix). Injury. 2001;32:63-66.

13. Wenzl ME, Porte T, Fuchs S, et al. Delayed and non-union of the humeral diaphysis-compression plate or internal fixator? Injury Int $J$ Care Injured. 2004;35:55-60.

14. Ring D, Kloen P, Kadzielski J, et al. Locking compression plates for osteoporotic nonunions of the diaphyseal humerus. Clin Orthop Relat Res. 2004:425:50-54.

15. Kääb MJ, Frenk A, Schmeling A, et al. Locked internal fixator: sensitivity of screw/plate stability to correct insertion angle of the screw. J Orthop Trauma. 2004; 18:483-487.

16. Zimmerman MC, Waite AM, Deehan M, et al. A biomechanical analysis of four humeral fracture fixation systems. J Orthop Trauma. 1994;8: 233-239. 